\title{
Les systèmes produit-service (SPS) : leviers de la transition des économies industrielles
}

\section{Product-service systems as transition enablers for industrial economies}

\author{
Blandine Laperche ${ }^{1}$, Céline Merlin-Brogniart ${ }^{2}$ \\ ${ }^{1}$ Réseau de Recherche sur l'Innovation, Université du Littoral Côte d'Opale, blandine.laperche@univ-littoral.fr \\ ${ }^{2}$ Réseau de Recherche sur l'Innovation, Université de Lille, celine.merlin@univ-lille.fr
}

RÉSUMÉ. Cet article introduit le numéro spécial de Technologie et Innovation sur les systèmes produit-service (SPS). II revient sur l'origine du terme, présente son actualité par le biais de quelques éléments bibliométriques et introduit les principaux débats actuels. Les systèmes produit-service illustrent la tertiarisation des économies industrielles, et mettent l'accent sur la complémentarité entre les biens et les services. Si le développement de services par l'industrie est un phénomène relativement ancien, elle s'accroit actuellement pour des raisons combinant raisons économiques et environnementales. Cette nouvelle actualité se lit dans la forte progression d'articles publiés utilisant ce terme à partir du début des années 2000. Trois thèmes essentiels caractérisent les débats actuels: les retombées environnementales des SPS (économie circulaire), le lien entre SPS et économie de (la) fonctionnalité ${ }^{1}$ et les conditions de production et de diffusion des SPS. C'est dans ceux-ci que s'inscrivent les contributions de ce numéro spécial.

ABSTRACT. This article introduces Technologie et Innovation's special issue on product-service systems (PSS). It examines the origin of the term, presents its topicality through some bibliometric elements, and introduces the main current debates. Product-service systems illustrate the tertiarization of industrial economies, and emphasize the complementarity between goods and services. While the development of services by industry is a relatively old phenomenon, it is currently increasing for economic and environmental reasons. This new topicality can be observed in the significant increase in the number of articles published using this term since the early 2000s. Three main themes characterize the current debates: the environmental impacts of SPS (circular economy), the link between SPS and the functional economy, and the conditions for the production and dissemination of SPS. This special issue contributes to these debates.

MOTS-CLÉS. système produit-service (SPS), tertiarisation, économie circulaire, économie de la fonctionnalité, innovation, bibliométrie.

KEYWORDS. product-service system (PSS), tertiarization, circular economy, functional economy, innovation, bibliometrics.

La tertiarisation est un fait majeur du XXe siècle qui, après les révolutions industrielles, annonce l'émergence d'économies où les services représentent une part croissante de l'emploi et du produit intérieur brut. De multiples raisons/thèses expliquent cette tertiarisation des économies [GAD, 92]. Pour certains comme D. Bell [BEL 73], la société post-industrielle dominée par les services, résulte de la loi d'Engel, qui stipule que le pouvoir d'achat s'accroissant, la demande des consommateurs passe de biens primaires répondant à des besoins de nécessité, à des biens secondaires fournis par l'industrie, puis à des biens supérieurs, constitués de services. Associée à une productivité reconnue

\footnotetext{
1 II existe plusieurs approches de l'économie de fonctionnalité. Certains auteurs utilisent le terme " d'économies de fonctionnalité » pour englober ces approches. Une première approche, plutôt dans l'esprit du concept développé par Stahel de « Functional Economy ", s'intéresse à la fois aux échanges marchands et aux coordinations non-marchandes [BUC 05] [BOU 05] [VAN 14]. L'approche de l'économie de la fonctionnalité et de la coopération a été proposée essentiellement en France, à partir du début des années 2000 [TER 07] [ZAC 07]. Selon Sidoli [SID 17], la notion de solution territorialisée caractérise cette approche. Elle s'inscrit dans une perception plus large du développement durable incluant la transformation et le bien-être au travail.
} 
plus faible dans les services, cette demande accrue renforce l'emploi dans les activités de services. D'un autre côté, les conceptions néo-industrielles considèrent la croissance des services en partant de l'industrie, qui est cœur de l'activité économique. Les services seraient ainsi un mal nécessaire, ou une éponge en cas de crise industrielle, et dans tous les cas leur croissance ou leur diminution serait dépendante des évolutions de l'industrie. On retrouve dans ces analyses les approches d'inspirations marxistes qui associent principalement la production de la valeur à l'industrie, mais aussi les approches keynésiennes et régulationnistes. Entres les deux approches, des analyses sur la complémentarité entre biens et services sont également développées [BEA 81], mettant l'accent sur la complexification de la production et des besoins, qui justifie la part croissante de la production des services.

Cette complémentarité entre biens et services fait suite aux phénomènes inverses d'industrialisation des services et de "servitization » des biens qui a contribué à rendre floue la distinction entre biens et services. L'industrialisation des services est ancienne, déjà décrite par Levitt en 1976 [LEV 76]. Elle consiste en l'application de méthodes de production industrielle aux organismes de service. Les entreprises et les organisations de service ont suivi le même processus d'innovation que l'industrie, par la recherche de mécanisation croissante, et de gains de productivité. Ce phénomène est encore à l'œuvre aujourd'hui.

La « servitization» (ou servicisation) de l'offre de biens consiste, à l'inverse, à transformer l'offre de biens en une offre de services. Ce phénomène est historiquement consécutif au premier. Néanmoins, il est aussi dans certaines de ces formes, relativement ancien puisque l'ajout de services à une offre de biens pour en améliorer la qualité ou se différencier des autres entreprises est propre aux économies fordistes [FUR 97, FUR 10]2. Le phénomène peut aussi être appréhendé à des périodes plus anciennes. En prenant l'exemple d'une entreprise de l'industrie lourde fondée au cours du XIXe siècle, Bryson [BRY 10] identifie les différents types de services et leur importance dans l'activité de cette entreprise. Cependant, depuis les années 1990, il y a eu selon l'auteur un changement dans la relation entre les services et les intrants manufacturiers dans le processus de production qui prend la forme de la création de systèmes de production hybrides et de produits hybrides qui mélangent processus de fabrication et de services.

L'offre intégrée de produits-services est aujourd'hui analysée par de nombreux auteurs qui emploient différents termes pour retranscrire ce phénomène [DJE 17] [SER 18] : les " complex packages » [BRE 88], les «product-service bundles » [VAN 88, STR 02] les «hybrid products » [SHA 09] ou « hybrid offerings » [ULA 11], les ventes fonctionnelles « Functional sales » [LIN 01], ou encore les Product-Service Systems [GOE 99, MON 02, BAI 07]. A partir du milieu des années 1990, c'est le terme Product-Service Systems (PSS) qui va se généraliser. Ce terme rassemble les stratégies visant «à offrir au lieu d'un produit ou d'un service seul, un ensemble intégré de produits et de services » [VAN $07: 7]$.

Ces offres de solutions conduisent à des réflexions sur de nouveaux modèles théoriques qui proposent de définir les produits (que ce soit un bien ou un service) par leurs fonctions (autrement dit, par les besoins qu'ils satisfont ou les services qu'ils rendent ${ }^{3}$ ). C'est le cas des économies de fonctionnalités [STA 97] dans lesquelles le consommateur n'acquiert plus la propriété d'un bien, mais l'usage de ce bien. D'autres approches telles que le Service-Dominant Logic [LUS 06] définissent la valeur par la valeur d'usage (« valeur-in-use »), permettant de ne plus faire de différence entre les biens et les services. D'après les travaux de Lancaster [LAN 66] et suivant l'exemple de Saviotti et Metcalfe [SAV 84], l'approche en termes de caractéristiques définit elle

\footnotetext{
${ }^{2}$ Certaines firmes industrielles se sont tellement transformées qu'elles ont basculé dans l'univers des services (ex IBM [AHA 13])

${ }^{3}$ Ex : Le besoin de mobilité, de sécurité, les enjeux de santé, d'éducation
} 
aussi un produit (bien ou service) comme un ensemble de caractéristiques de services [GAL 97]. Ces travaux s'inscrivent dans une perspective « intégratrice » des biens et des services [GAL 94].

\section{Le développement des systèmes produit-service : de la raison économique à la raison environnementale}

Le développement des SPS pourrait tant s'expliquer par la complexité technologique qui nécessite de fournir des services supplémentaires aux consommateurs, que par la nécessité de se différencier dans une concurrence accrue et de s'approcher au plus près des besoins, voire des émotions des consommateurs pour les transformer en marchandises ${ }^{4}$. Les premières raisons évoquées pour expliquer l'émergence de ces ensembles produit-service sont en effet des raisons purement économiques, associées à la rentabilité et à l'obtention d'un avantage concurrentiel [BAI 10, FUR 97, FUR 10].

Le développement des systèmes produit-service traduit aussi les formes complexes que prend aujourd'hui l'innovation. Longtemps étudiée par commodité sous l'angle de l'innovation technologique (la plus visible, la plus facilement mesurable), la définition contemporaine de l'innovation élargit l'approche tout en segmentant/distinguant bien les formes de l'innovation. C'est le cas dans la définition pionnière de Schumpeter [SCH 11] ou de la définition proposée dans le manuel d'Oslo (2005) [LAP 18]. Mais dans la réalité, les formes de l'innovation - de produit, de procédé, d'organisation, de commercialisation- sont souvent reliées ou associées. Ainsi, dans la communication des entreprises, il est courant d'entendre ou de lire que celles-ci proposent des « solutions ", qui combinent différentes formes de l'innovation (produit, procédé, organisation, commercialisation). Ces innovations font aussi la part belle aux services qui peuvent prendre la forme de l'innovation de produit (par exemple, un nouveau service bancaire), d'organisation (une méthode de travail innovante) ou de commercialisation (un service de vente à distance).

Le développement de services associés aux produits peut également être lié à la volonté de satisfaire un besoin non ou mal satisfait. Par exemple, ils peuvent être liés à la prise en charge des individus, à l'instar des "géront'innovations », les innovations à destination des personnes dépendantes, qui associent souvent produits et services [LAP 19]. Dans ce dernier cas, ces solutions sont plutôt développées dans l'optique de fournir une offre complète et diversifiée de produits et de services, adaptée aux besoins de chacun des consommateurs.

Mais le développement systèmes produit-service connaît une nouvelle actualité dans le contexte de la réflexion sur la transition des économies et de l'industrie vers un mode de fonctionnement orienté vers le développement durable. Ces «solutions » proposées par les entreprises manufacturières comme ArcelorMittal, STMicroelectronics, Saint-Gobain et Schneider Electric, Renault combinent des produits, des composants et des services afin d'adapter l'offre aux besoins des consommateurs, lesquels sont liés aux contraintes environnementales de plus en plus fortes [LAP 13]. Le terme même de « système produit-service » est défini en insistant sur sa dimension environnementale (en termes d'impacts plus faibles sur l'environnement). Le terme Système Produit-Service (SPS) a été défini par Goedkoop et al. [GOE 99] comme « un ensemble de produits et de services capables de satisfaire conjointement les besoins d'un utilisateur et fournis par une entreprise ou par une alliance stratégique ». Dans ce rapport, les auteurs insistent sur les avantages environnementaux qui peuvent être associés à cette offre. Dans les définitions qui suivent, les chercheurs mettent en avant l'association de produits, de services, de réseaux d'acteurs et d'infrastructures d'appui qui s'efforce en permanence d'être compétitive, de satisfaire les besoins des

\footnotetext{
${ }^{4}$ Selon la thèse de E. Illouz (2019), Les marchandises émotionnelles, Editions premier parallèle, Paris.
} 
clients et d'avoir un impact environnemental inférieur aux modèles économiques traditionnels. Selon la revue de littérature de Baines et al. [BAI 07], la plupart des auteurs ont, dans les grandes lignes, adopté les éléments clés de cette définition. Le lien avec l'environnement réside dans le fait de passer de la vente d'un produit à la vente d'une fonction capable de satisfaire les besoins des consommateurs tout en réduisant l'impact environnemental [MON 02, TUK 15]. C'est le fait de devoir rendre durables les équipements utilisés, la dématérialisation et la part croissante des services dans l'offre qui permettent de réduire l'impact environnemental de l'activité. Cette approche peut être reliée à la notion d'économie de fonctionnalité qui vise à "optimiser l'utilisation (ou la fonction) des biens et services et donc la gestion des richesses existantes (biens, connaissances et nature). L'objectif économique de l'économie de fonctionnalité est de créer la valeur d'usage la plus élevée possible le plus longtemps possible tout en consommant le moins possible de ressources matérielles et d'énergie" [STA 06] [STA 97]. Les systèmes produit-service possèdent certaines caractéristiques, bien résumées par Geum et Park [GEU 11] : Les SPS intègrent des produits et des services, combinent la création de valeur et la performance environnementale (mesurée par la réduction de l'impact environnemental), et ils impliquent une transition vers l'économie de (la) fonctionnalité (la propriété du bien n'est plus transférée au consommateur mais plutôt conservée par le producteur).

Selon la part de produit ou de service qui compose le SPS, différents types de SPS peuvent être définis, associés à des potentiels de durabilité eux-aussi variés : on distingue ainsi les services orientés produits (Les services sont simplement ajoutés à un système de produits existant afin de garantir la fonctionnalité et la durabilité du produit détenu par le consommateur), les services orientés usage (Les services intensifient l'usage d'un produit. L'utilisation ou la disponibilité d'un produit est vendue mais le produit n'est pas la propriété du consommateur : location de produit, partage, mise en commun) et les services orientés résultats. Ces derniers sont les seuls vrais SPS orientés vers les besoins. Un résultat ou une capacité est vendue à la place d'un produit. L'entreprise et ses partenaires deviennent responsables de tous les coûts liés à l'obtention d'un résultat et ainsi, sont fortement incités à optimiser l'usage des matériaux et l'énergie [TUK 04, TUK 06, GEU 10].

\section{Quelques éléments bibliométriques}

Une recherche sur le portail Scopus à partir du mot clé product-service-system (PSS) fait apparaitre sur la période 1983 - 2018, 1926 documents comportant cette expression dans le titre, le résumé ou les mots clés. Ces documents sont en grande partie des actes de conférences $(50,6 \%)$ et des articles de revues $(37,9 \%)$. Nous pouvons remarquer sur le graphique 1 , que mis à partir un article paru en 1983 comportant ce mot clé, les publications sur ce thème s'accroissent fortement à partir des années 2000, en lien avec cette nouvelle composante « environnementale » associée à cet ensemble de produits et de services.

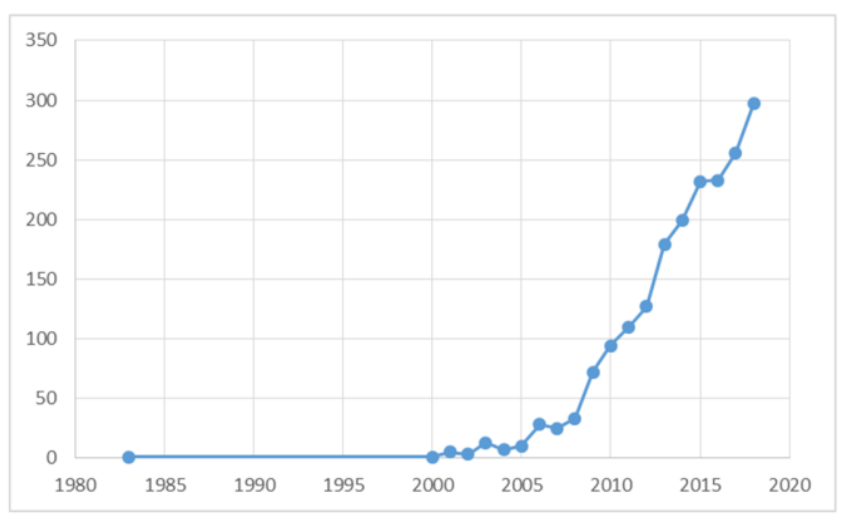

Graphique 1. Evolution des publications internationales sur les "systèmes produits-services " (1983-2018) 
Source : Source Scopus, documents comportant le terme «product-service-system » dans le titre, le résumé ou les mots clés. Réalisé le 2 août 2019, sur la période 1983 (première date répertoriée par Scopus) 2018, soit 1926 documents.

Il faut noter que la recherche sur ce thème est essentiellement internationale. La même recherche sur le portail Cairn en France, à partir du mot clé « système produit-service » ne fait apparaître que 5 occurrences (à partir de 2013 seulement) dans les revues suivantes ; Innovations, Revue d'économie et de management de l'innovation (2, en 2015 et 2017), Marché et organisations (1 en 2013), Annales des Mines (1 en 2014) et Revue française de gestion (1 en 2016). Certes, ce portail est spécialisé dans les sciences humaines et sociales, qui constituent une minorité des publications sur ce thème. Mais cela montre la relativement faible diffusion de ce concept et de la littérature qui s'y associe en France, que cherche en partie à combler ce numéro spécial sur les SPS.

Les SPS ont été analysés dans différentes disciplines en particulier dans les sciences de l'ingénieur, dans les réflexions sur la place dans laquelle l'entreprise se situe au sein de la chaine de valeur, et en sciences sociales, afin de trouver une réponse pour élargir l'offre de l'entreprise face à la restriction des débouchés. Le poids important des disciplines d'ingénierie et d'informatique se lit dans le graphique 2. L'expression Système produit-service apparait le plus (dans les titres, résumés, mots clés) dans les publications en ingénierie qui représentent $37 \%$ du total sur la période considérée, les sciences informatiques $15 \%$, les disciplines de management, comptabilité auxquels on ajoute l'économie comptent pour $16 \%$ du total, les sciences sociales seulement 3\%.

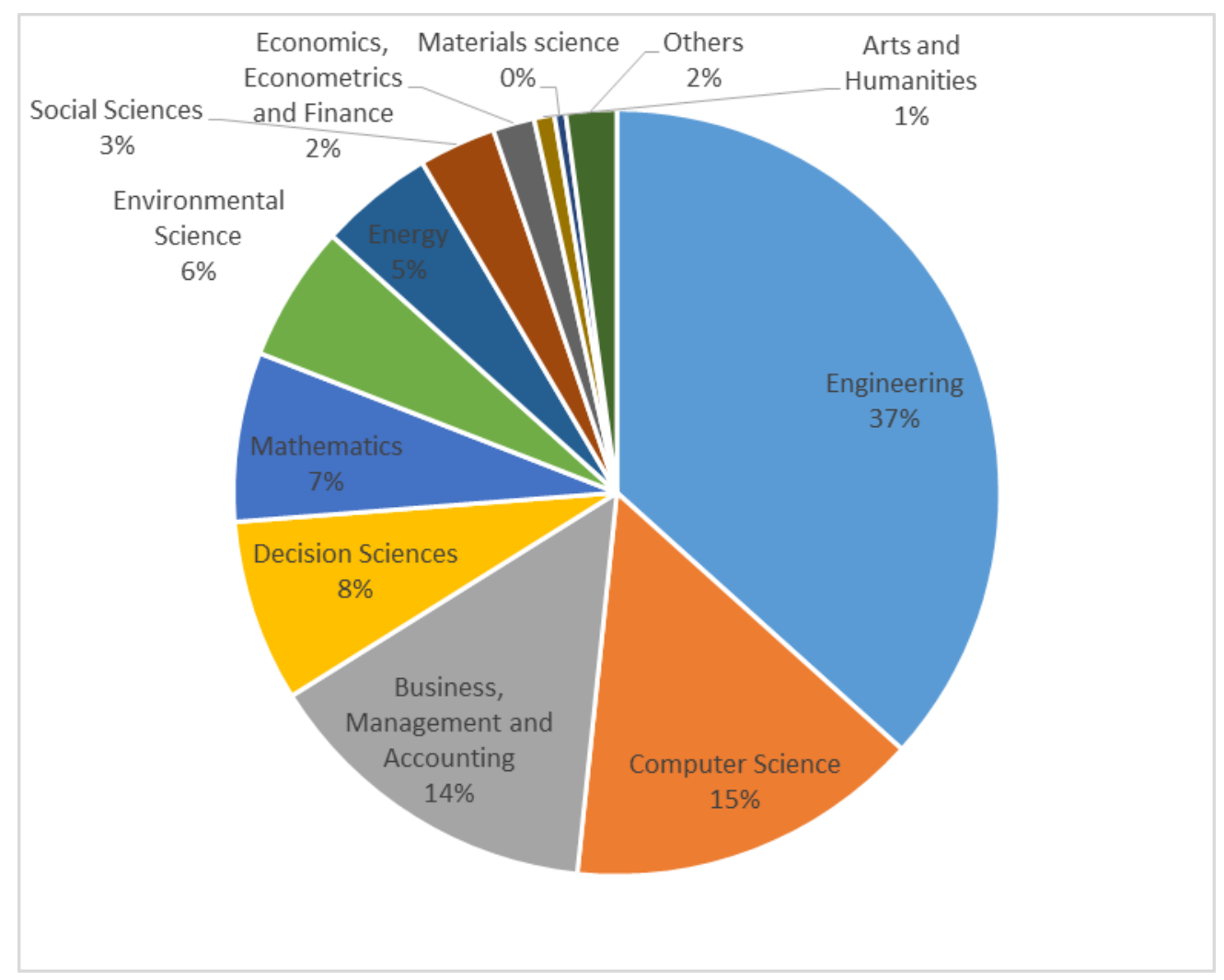

Graphique 2. Principaux domaines d'études/ disciplines des publications internationales sur les systèmes produit-service (1983-2018).

Source : à partir des mots clés apparaissant dans les titres, résumés et mots clés- données Scopus, sur la période 1983-2018. 
Les principaux supports de publication sont ainsi des revues d'ingénierie ou à tendance pluridisciplinaire (graphique 3) (c'est le cas de Journal of Cleaner Production par exemple).

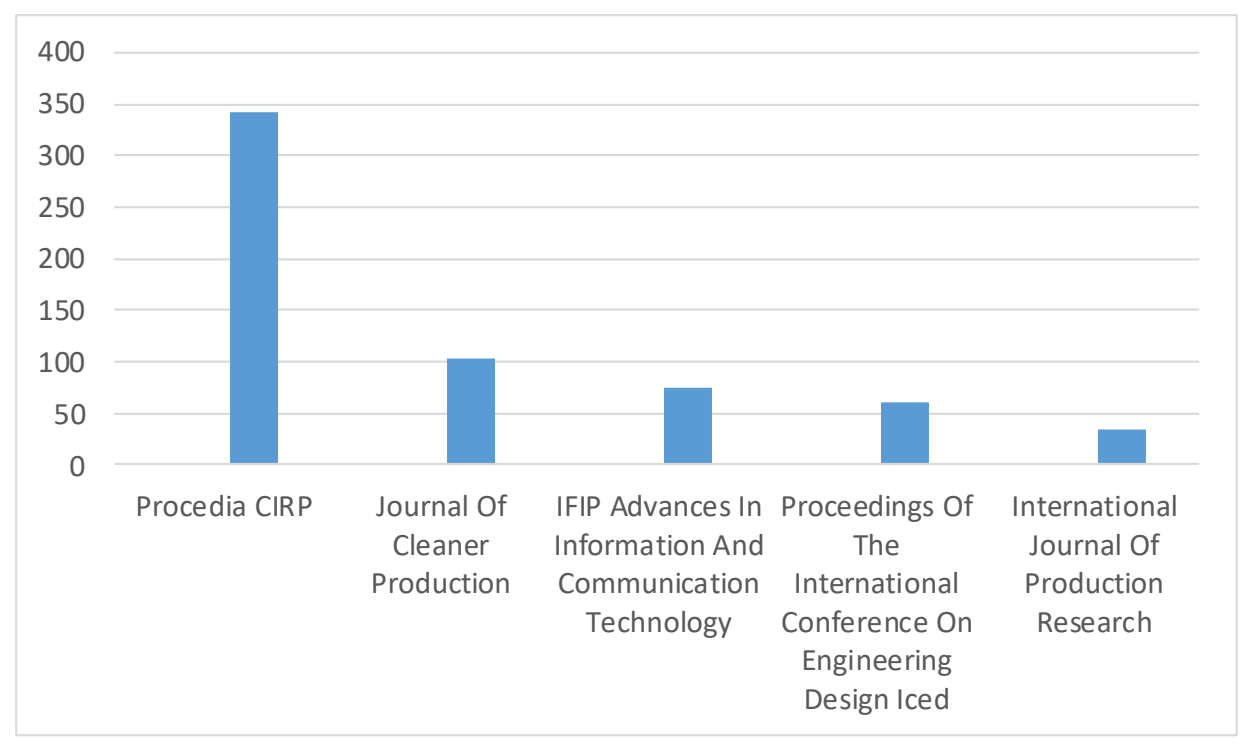

Graphique 3. Principaux supports de publications sur les systèmes produits services (1983-2018)

Sources : Les 5 premiers supports de publications sur le thème des SPS, données Scopus, sur la période 1983- 2018.

\section{Principaux thèmes actuels de recherche autour des SPS}

La lecture plus qualitative des publications nous amène à mettre en avant les principaux thèmes et questionnements qui irriguent la recherche sur les systèmes produit-service. Nous proposons trois thèmes fondamentaux (les retombées environnementales des SPS, le lien entre SPS et économie de (la) fonctionnalité, les conditions de production et de diffusion des SPS). Ces thèmes sont ceux dans lesquels s'intègrent les articles qui composent ce numéro spécial, contribuant ainsi aux débats actuels.

Les SPS, comme dit précédemment, ont des objectifs variés, l'objectif de réduction de l'impact environnemental peut être dominant, dans d'autres cas, l'objectif de différenciation de la concurrence et de réponse aux besoins de catégories de consommateurs sera le premier argument. En particulier, la question qui se pose est celle des impacts de l'offre de SPS : sont-ils toujours efficaces en termes de retombées économiques et environnementales ? Si en théorie, les résultats devraient être positifs, le décalage avec la réalité est parfois manifeste [PAR 14]. Les travaux récents mettent l'accent comme le font dans ce numéro Angeles Perreira et Xavier Vence sur le lien entre SPS et économie circulaire. L'économie circulaire est également un concept récent et non encore stabilisé [KOR 18]. Il implique de dépasser l'économie traditionnelle fonctionnant sur le mode « linéaire » Extraire - Produire - Consommer - Jeter pour aboutir à un fonctionnement plus circulaire fondé des boucles de réutilisation/transformation destinées à réduire l'impact environnemental final de l'activité économique [BOL 19, GAL 16]. L'article de Angeles Pereira et Xavier Vence analyse la contribution des business models basés sur les SPS à la mise en œuvre de l'économie circulaire. Un examen critique de la littérature de la fin des années 1980 à nos jours montre que peu d'articles proposent des exemples concrets de l'amélioration de l'éco-efficacité des modèles de PSS. Une perspective systémique et critique de ces business models SPS permettrait de mieux évaluer leur contribution aux principes de l'économie circulaire. 
Le lien entre système produit-service et économie de (la) fonctionnalité est également un thème fondamental. Nous avons vu en effet plus haut que selon la part de service incluse dans le système produit-service, le basculement vers une économie de (la) fonctionnalité est plus ou moins envisageable. Dans tous les cas, les SPS s'inscrivent dans les mutations des formes de l'échange. Dans leur article, Benjamin Serra et Nicolas Buclet proposent de différencier les différents concepts basés sur l'économie des usages, en lien avec «l'économie de fonctionnalité », à partir du modèle des cités de L. Boltanski et L. Thévenot [BOL 91]. Les auteurs étudient la manière dont ces différents concepts remettent en cause les principes industrialo-marchands du système économique. Certaines conceptions de l'économie de fonctionnalité ne remettent pas fondamentalement en cause les formes d'échange, mais infléchissent ces formes d'échanges de sorte à en améliorer la performance, en termes économiques, sociaux et environnementaux.

Le lien entre système produit-service et les économies de fonctionnalité peut aussi être analysé à partir de leur caractère innovant. Céline Merlin-Brogniart examine la dynamique de l'innovation des différents modèles économiques décrits par les différentes approches des économies de fonctionnalité. Il est possible de repérer la nature et la dynamique de l'innovation de ces modèles à différentes échelles d'analyse (micro, méso, macro). Cet article suggère qu'un grand nombre de développements pratiques des économies de fonctionnalité existe, en fonction du nombre d'acteurs et de l'échelle spatiale dans laquelle ces modèles s'inscrivent. La dynamique d'innovation repose essentiellement sur l'intégration d'une logique servicielle, mais l'intensité de l'innovation est plus ou moins importante en fonction du degré de rupture de ces modèles économiques avec le modèle de croissance matérielle intensive.

Une troisième thématique prégnante dans les débats actuels sur les systèmes produit-service est celle des conditions de la production et de la diffusion des SPS. Du côté de la demande, pour faciliter la diffusion des systèmes produit-service, il est nécessaire de bien connaître les motivations des consommateurs/utilisateurs. Les SPS prennent de multiples formes dont celle de l'économie du partage. Dans ces modèles, les utilisateurs sont essentiels pour la coproduction du service. Ils sont à l'origine de la production de valeur. Marcos Lima, Patricia Baudier, Karim Alawamleh, Bjorn Seguin analysent les motivations des utilisateurs des plateformes de co-voiturage, en explorant les facteurs intrinsèques et extrinsèques qui expliquent leur attitude et leur comportement d'adoption de ces systèmes. Ils mettent en évidence le fait que deux facteurs intrinsèques (le sentiment écologique et le plaisir) et un des facteurs extrinsèques (l'avantage économique) contribuent fortement à créer une attitude favorable en vue de cette pratique. A l'inverse, d'autres motivations, notamment les attentes en matière de réputation, ne semblent pas influencer les attitudes ou l'intention comportementale de l'utilisateur.

Du côté de l'offre, les caractéristiques systémiques des SPS doivent en effet être prises en compte. Quelles relations entre systèmes produits-services et open innovation, donc quel est le rôle des partenariats, des réseaux dans leur mise en œuvre ? En effet, « un SPS est une construction sociale », pour reprendre l'expression de Morelli [MOR 06, p. 1496]. La définition même d'un service, au-delà de ses caractéristiques courantes (intangibilité, immatérialité), nécessite une interaction avec l'utilisateur. L'intégration de produits et de services dans les SPS et l'offre de solutions requièrent ainsi des partenariats avec différentes parties prenantes, des consommateurs et des fournisseurs de certains composants du SPS. Le but est tant de réduire les coûts de développement que d'obtenir des compétences complémentaires. Dans ce numéro, Clément Morlat et Jean-Marc Douguet expliquent comment le portail internet contributif ( ePLANETe.Blue ») mobilisé lors du programme ANR «AGREGA » a permis d'accompagner les acteurs de la filière d'approvisionnement de la Région Ile-de-France en granulats de construction nécessaires au GrandParis vers une représentation collective des interactions permettant de se diriger vers l'économie circulaire. Ces acteurs de la filière «approvisionnement en granulats » ont ainsi co-construit une 
méthodologie d'évaluation ad hoc destinée à favoriser un couplage structurel entre deux types de SPS.

Dans une approche plus centrée sur la firme, Sophie Peillon analyse les logiques d'innovation sous-jacentes au développement de systèmes produit-service (SPS) par les entreprises industrielles. Selon cette auteure, le développement de SPS orientés produit, usage ou résultat repose sur des logiques différenciées en termes d'exploitation et d'exploration. Trois stratégies sont repérables : une stratégie d'exploitation, une stratégie d'exploration ou une forme d'ambidextrie organisationnelle. Cet article montre que plus le changement de business model sous-tendu par la servicisation est important, plus il repose sur une stratégie d'exploration.

Parmi les thèmes traitant des conditions du développement de l'offre des SPS, les recherches en ingénierie s'intéressent aux méthodologies permettant de concevoir ou d'élaborer la planification stratégique du développement des SPS [MOR 06, GEU 10, GEU 11, AUR 10]. Les méthodologies de conception ont pour objet de fournir des outils pour la planification, la prévision et la gestion pas à pas des SPS. Dans ce numéro, l'article de Florence Bazzaro et Yann Meyer propose une approche méthodologique, adaptée aux chercheurs de tout domaine thématique, leur permettant de s'interroger, de mener une réflexion sur les enjeux sociaux et environnementaux liés aux technologies, aux produits, aux processus, objets de leurs études. Ainsi, la problématique de recherche s'interroge sur quelles méthodes, outils, démarches de conception pour guider le chercheur dans la prise en compte des dimensions environnementales et sociales lors du développement d'une brique technologique. Cette méthodologie peut très bien s'appliquer à une brique technologique-service, donc à un SPS comme le soulignent les auteurs.

Au final, les contributeurs de ce numéro spécial mettent en avant la multiplicité des concepts associés à cette tertiarisation de l'économie : aux systèmes produit-service sont associés les concepts de servicisation, d'économie de (la) fonctionnalité, d'économie du partage, d'économie circulaire pour ne citer que les principaux. Ce foisonnement de la recherche sur les nouveaux modèles de production et d'échange montre les limites de la production et de la consommation matérielle intensive qui ont caractérisé les décennies et siècles qui nous précèdent (voir sur ce point [DAU 18]). Il montre aussi la recherche tant théorique qu'empirique de nouveaux leviers pour favoriser la transition des économies. Cette transition peut toutefois prendre des voies différentes, que ce soit vers un renouveau de la production et de la consommation dans un système dont les bases de fonctionnement sont inchangées, ou encore vers la mutation de ce système afin d'atteindre un mode de fonctionnement plus respectueux en particulier de l'environnement naturel. Il faut reconnaître que sur ce point les visions sont assez peu tranchées, voir plus confuses... Les auteurs de ce numéro spécial nous invitent ainsi à une analyse critique des concepts analysés en particulier des systèmes produit-service et à une approche systémique de ceux-ci ainsi que de notion même de transition [PIC 17], combinant différent niveaux d'analyse (micro, méso et macro), afin d'en faire de véritables leviers d'innovation radicale.

\section{Bibliographie}

[AHA 13] Ahamed Z., InOHARA T. Kamoshida A, "The Servitization of Manufacturing: An Empirical Case Study of IBM Corporation”, International Journal of Business Administration, 4, n² 2, 2013.

[AUR 10] AURICH J.C., MANNweILER C., SCHWEITZER E., "How to design and offer services successfully", CIRP Journal of Manufacturing Science and Technology, no. 2, p. 136-143, 2010.

[BAI 07] BAINES T.S., LightFOOT H., STEVE E. et al., "State-of-the-art in product-service systems", Part B: Journal of Engineering Manufacture, Proc. IMechE, vol. 221, 2007. 
[BAi 10] Baines, T.S., Lightfoot, H., Benedettini, O., Whitney, D., KAY, J.M., "The adoption of servitization strategies by UK-based manufacturers”, Part B: Journal of Engineering Manufacture, Proc IMechE vol. 224, 2010.

[BEA 81] Bearse P., Karasek R., Noyelle T., Stanback T., Services, The new Economy, Allanhed and Osmun, Totowa N.J., 1981.

[BEL 73] BeLl D., The Coming of Post-Industrial Society, a Venture in Social Forecasting, Basic Books, New York, 1973.

[BOL 91] BoltANSKi L., TheVENOT L., De la justification. Les économies de la grandeur, Gallimard, Paris, 1991.

[BOL 19] BOLDRINI J.C., ANTHEAUME N., "Une transition vers quelle économie circulaire ?», Technologie et Innovation, 2019, DOI : 10.21494/ISTE.OP.2019.0339, 2019.

[BOU 05] Bourg D., BuClet N., «L'économie de fonctionnalité : changer la consommation dans le sens du développement durable », Futurible, n³13, p. 27-37, 2005.

[BRE 88] BRESSAND A., NicOlAÏDIS K., «Les services au cœur de l'économie relationnelle », Revue d'économie Industrielle, $\mathrm{n}^{\circ} 43,1 \mathrm{er}$ trimestre, p. 141-163, 1988.

[BUC 05] BUCLET N., "Concevoir une nouvelle relation à la consommation : l'économie de fonctionnalité », Annales des Mines, Vol.39, juillet, p.57-66, 2005.

[BRY 10] BRYSON, J.R., Service Innovation and Manufacturing Innovation: Bundling and Blending Services and Products in Hybrid Production Systems to Produce Hybrid Products, In: Gallouj, Djellal (Ed.), The Handbook of Innovation and Services. A Multi-Disciplinary Approach, E. Elgar, Cheltenham, UK.pp. 679- 721, 2010.

[DAU 18] DAUMAS, J.C., La révolution matérielle. Une histoire de la consommation, France XIXe-XXe siècle, Flammarion, Paris, 2018

[DJE 17] DJEllal F., Gallouj F., Quinze avancées et quinze défis pour la recherche sur l'innovation dans les services", Séminaire Maghtech, 24 janvier 2017

[FUR 97] FURRER, O., «Le rôle stratégique des services autour de produits », Revue Française de Gestion 113, p. 98108, 1997.

[FUR 10] FURRER, O., "A Customer Relationship Typology of Product Services Strategies”, dans Gallouj, Djellal (Ed.), The Handbook of Innovation and Services. A Multi-Disciplinary Approach, E. Elgar, Cheltenham, UK., p. 679- 721, 2010.

[GAD 92] GADREY J., L'économie des services, Repères, La Decouverte, Paris, 1992.

[GAL 16] GALLAUD D., LAPERCHE B., Economie circulaire et développement durable, Iste, Londres, 2016.

[GAL 94] GALLOUJ F., Economie de l'innovation dans les services, l'Harmattan, Logiques Economiques, 1994.

[GAL 97] GALlOUJ F., WeInStEIn O., “Innovation in services”, Research Policy, vol. 26, p. 537-556, 1997.

[GEU 10] GEUM Y., PARK Y., "Development of technology Roadmap for Product-service systems (TRPSS)". Proceedings of the 2010 IEEE IEEM, pp. 410-414, Macao China, 2010.

[GEU 11] GEUM Y., PARK Y., "Designing the sustainable product-service integration: a product-service blueprint approach", Journal of Cleaner Production, vol. 19, p. 1601-1614, 2011.

[GOE 99] GoedKoop C., VAN HALER H., Rielete Rommers P., "Product service-systems, Ecological and Economics basics", Report for Dutch Ministries of Environment (VROM) and Economic affairs (EZ), 1999.

[KOR 18] Korhonen J., Honkasalo A., SePPÄLÄ J., "Circular Economy. The concept and its limitations", Ecological Economics, 143, p. 37-48, 2018.

[OCD 05] OCDE, Manuel d'Oslo. Principes directeurs pour le recueil et l'interprétation des données sur l'innovation, 3e édition, OCDE, Paris, 2005.

[LAN 66] LANCASTER K.J., "A New Approach to Consumer Theory”, Journal of Political Economy, 14, p. 133-156, 1966.

[LAP 13] LAPERCHE B., PICARD F., "Environmental constraints, product-service systems development and impacts on innovation management: learning from manufacturing firms in the French context", Journal of Cleaner Production, vol. 53, p. 118-128, 2013. 
[LAP 19] LAPERChe B., Boutillier S., DJellal F, Ingham M., Liu Z., PiCARD F. Reboud S, TANGUy C. UZUNidis D., Innovating for elderly people: the development of geront'innovations in the French silver economy, Technology Analysis \& Strategic Management, Volume 31, - Issue 4, 462-476, DOI: 10.1080/09537325.2018.1520975, 2019.

[LAP 18] LAPERCHE B., Le capital savoir de l'entreprise, Iste, Londres, 2018.

[LEV 76] LEVITT, T., “The Industrialisation of Servive”, Harvard Business Review, 54, n5, p.63-74, 1976.

[LIN 01] LINDAHL M., OLundH G., "The meaning of functional sales", $8^{\text {th }}$ International Seminar on Life Cycle Engineering, Life Cycle Engineering: Challenges and Opportunities, p. 211-220, 2001.

[LUS 06] LUSCH, R, VARGO S, "Service-Dominant logic: reactions, reflections and refinements", Marketing Theory, 6, n³, p. 281-288, 2006.

[MON 02] MONT O., "Clarifying the Concept of Product-Service System", Journal of Cleaner Production, vol. 10, no. 3, p. 237-245, 2002.

[MOR 06] MORELLI N., "Developing new product service systems (PSS): methodologies and operational tools". Journal of Cleaner Production, vol. 14, p. 1495-1501, 2006.

[PAR 14] PARIDA V., SJOdin D.R., Wincent J., KohtamaKi M., Mastering the transition to product-service provision: Insights into business models, learning capacities and capabilities, Research Technology Management, vol.57, 3, p.44-52, 2014

[PIC 17] PICARD F., TANGUY C., Innovation et transition techno-écologique, Iste, Londres, 2017.

[SAV 84] SAVIOTTI P.P., METCALFE, "A theoritical approach to the construction of technological output indicators", Research Policy, 3, p. 141-151, 1984.

[SCH 11] SCHUMPETER J.A., Théorie de l'évolution économique. Recherche sur le profit, le crédit, l'intérêt et le cycle de la conjoncture 1911 (traduction française 1935), disponible sur : http://classiques.uqac.ca/classiques/Schumpeter_joseph/theorie_evolution/theorie_evolution.html

[SER 18] SERRA B., Perspectives durables et territoriales des économies de fonctionnalité légitimes. Une interprétation en termes conventionnalistes, Thèse dirigée par le Pr. BUCLET, Université Grenoble Alpes, 18 juin, 2018.

[SHA 09] ShankAR V, BERRY L, DOtZel T, "A practical guide to combining products and services", Harvard Business Review, November, p. 94-99, 2009.

[SID 17] SIDOLI Y., L'usage en partage : Analyse comparative des modèles socio-économiques d'"économie de (la) fonctionnalité" et d'"économie collaborative, Thèse Sociologie. Université Côte d'Azur, 2017. <NNT : 2017AZUR2008>. <tel-01516611>, 2017.

[STA 97] STAHEL W.R., The Functional Economy: cultural and organizational change from the industrial green game: implications for environmental design and management, National Academy Press, Washington, DC, 1997.

[STA 06] STAHEL W. R., The Performance Economy, Palgrave Macmillan, 2006.

[STR 02] STREMERCH, S., TELLIS G J, "Strategic Bundling of products and prices: A new synthesis for marketing", Journal of Marketing, 66, ${ }^{\circ} 1$, p. 55-72, 2002.

[TER 07] TERTRE C. DU, « Economie de la fonctionnalité, développement durable et innovations institutionnelles », dans E. Heurgon et J. Landrieu (dir), L'économie des services pour un développement durable, L'Harmattan, Paris, p.241-255, 2007.

[TUK 04] TUKKeR A., HINES F., MARIN O. (eds)., "Eight types of product-service system: eight ways to sustainability? Experiences from SusProNet", Business Strategy and the Environment, vol. 13, no. 4, p. 246-260, 2004.

[TUK 06] TUKKER A., TISCHNER U., "Product-services as a research field: past, present and future, reflections from a decade of research". Journal of Cleaner Production, vol. 14, p. 1552-1156, 2006.

[TUK 15] TUKKER A, "Product services for a resource-efficient and circular economy - A review", Journal of Cleaner production, 97, p.76-91, 2015

[Ula 11] Ulaga W., Reinartz, WJ, "Hybrid Offerings: How Manufacturing Firms Combine Goods and Services Successfully", Journal of Marketing, 75, nº, p. 5-23, 2011. 
[VAN 88] VANDERmerwe S, RADA J, "Servitization of Business: Adding Value by Adding Services", European Management Journal, 6 (4), p. 314-324, 1988.

[VAN 07] VAN NIEL J., L'économie de fonctionnalité : définition et état de l'art, note de synthèse réalisée dans le cadre du groupe de travail "Economie de fonctionnalité" de la Commission Nationale du développement durable, mai, 2007

[VAN 14] VAN NIEL J., « L'économie de fonctionnalité : principes, éléments de terminologie et proposition de typologie », Développement durable et territoires, Vol.5 (1), [En ligne], 2014.

[ZAC 07] ZACKLAD M., " L'économie de la fonctionnalité encastrée dans la socio-économie des transactions coopératives : dynamique servicielle et filiation soutenable », dans E. Heurgon et J. Landrieu (dir), L'économie des services pour un développement durable, L’Harmattan, Paris, p.273-288, 2007. 